\title{
FUNCIONALIDADE DE MEMBRO SUPERIOR E QUALIDADE DE VIDA DE MULHERES COM CÂNCER DE MAMA SUBMETIDAS A TRATAMENTO FISIOTERAPÊUTICO
}

\author{
UPPER LIMB FUNCTIONALITY AND QUALITY OF LIFE OF WOMEN WITH BREAST CANCER \\ UNDERGOING PHYSIOTHERAPEUTIC TREATMENT
}

\section{Luana Paula Navaa ${ }^{a}$, Cibeli Ferreira Martins ${ }^{b}$, Simone Larac ${ }^{c}$, Fernanda Vargas Ferreira ${ }^{d}$}

aluanapn10@yahoo.com.br, bcibeli_martins@yahoo.com.br, cslarafisio@yahoo.com.br, danandafvf@gmail.com Universidade Federal do Pampa - Uruguaiana (RS), Brasil

\section{RESUMO}

Introduçáo: $\mathrm{O}$ câncer de mama é um grave problema de saúde pública, sendo a maior causa de morte feminina no Brasil. O tratamento pode ser cirúrgico e/ou conservador, podendo provocar linfedema e redução da amplitude de movimento do membro superior, principalmente nos movimentos de flexão e abdução de ombro, repercutindo negativamente na qualidade de vida. Objetivo: Verificar o impacto da aplicação de um protocolo fisioterapêutico sobre a funcionalidade e qualidade de vida de mulheres que foram submetidas ao tratamento do câncer de mama. Materiais e métodos: Estudo experimental do tipo antes e depois, composto por 10 sessôes de mobilização cicatricial, alongamentos, exercícios ativos livres em todos os planos de movimento em mulheres submetidas a tratamento cirúrgico e adjuvante, na faixa etária de 40 a 65 anos. Foram avaliadas quanto à amplitude de movimento (ADM), presença ou não de linfedema e qualidade de vida. Resultados: A amostra foi de 4 participantes com média de idade de $54 \pm 11,5$ anos, submetidas à mastectomia radical modificada e à quadrantectomia associada ou não à linfadenectomia axilar. Inicialmente, foram detectados déficits em todos os planos de movimento do ombro ipsilateral, destacando-se com menores graus de amplitude na flexão, extensão, abdução e rotaçáo externa, e após o protocolo observamos melhora da ADM em todos os movimentos do ombro, especialmente na flexão e abdução. Conclusão: Verificamos que um protocolo fisioterapêutico de curta duração melhorou a ADM de ombro e, consequentemente, sua qualidade de vida.

Palavras-chave: Câncer de mama; pós-operatório; amplitude de movimento; qualidade de vida; protocolo fisioterapêutico.

\section{ABSTRACT}

Introduction: Breast cancer is a serious public health problem and a major cause of female death in Brazil. Treatment can be surgical and/or conservativeand possible complications include lymphedema and reduced upper limb range of motion, particularly in shoulder flexion and abduction, reflecting negatively on the quality of life. Objective: Verify the impact of the application of a physical therapy protocol on functionality and quality of life of women with breast cancer. Materials and methods: An experimental (before and after) study design was used with a protocol composed of 10 sessions of scar mobilization, stretching, active-free exercises in all planes of motion in women aged between 40 and 65 years old subjected to surgery and adjuvant therapy. They were evaluated for range of motion (ROM), presence or absence of lymphedema and quality of life. Results: The sample consisted of 4 participants with mean age of $54 \pm 11.5$, who were subjected to modified radical mastectomy and to quadrantectomy associated or not to axillary lymphadenectomy. Initially, deficits were detected in all planes of ipsilateral shoulder's movement, noticing lesser degrees of amplitude in flexion, extension, abduction and external rotation. After the protocol, we observed ROM improvement in all shoulder movements, especially in flexion and abduction. Conclusion: It was observed that a short physiotherapeutic protocol promoted shoulder ROM improvement's and had a positive impact on the quality of life.

Keywords: Breast cancer; postoperative; range of motion; quality of life; physiotherapeutic protocol. 


\section{Introdução}

O câncer de mama (CM) apresenta-se como um grave problema de saúde pública em todo o mundo, sendo no Brasil a maior causa de óbitos na população feminina, principalmente na faixa etária de 40 a 69 anos $^{1}$. Constituise como a neoplasia de maior ocorrência entre mulheres de países desenvolvidos e em desenvolvimento ${ }^{2,3}$.

Conceitualmente, o câncer resulta de uma multiplicação desordenada de células, apresentando-se de diversas formas clínicas e morfológicas com potencial risco de metástase, por causa do extenso sistema linfático e da presença de gânglios na região do tronco superior ${ }^{4}$. Como fatores de risco ao $\mathrm{CM}$, citamos a hereditariedade, a nuliparidade, a menopausa tardia, a obesidade, o sedentarismo e a menarca precoce ${ }^{1}$, fatores que demonstram a complexidade dessa doença.

O diagnóstico do $\mathrm{CM}$ se baseia no exame clínico das mamas, de imagens e/ou laboratoriais, com vistas à precocidade, uma vez que, no Brasil, há uma tendência a uma descoberta tardia, o que repercute na escolha dos tratamentos e possíveis repercussóes físico-funcionais, favorecendo, por sua vez, altos índices de morbimortalidade $^{1}$. Dentre as principais complicaçóes pós-tratamento, se destacam o linfedema, que é o acúmulo de proteína no interstício em virtude de deficiência do sistema linfático pós-intervenção cirúrgica e o esvaziamento axilar e/ou radioterapia, que gera um aumento do volume do membro superior impactando a sua funcionalidade 5 . Também outra limitação ocorre na amplitude de movimento do membro superior, especialmente na abdução e flexão, refletindo negativamente na realização das atividades de vida laborais e de lazer ${ }^{6}$.

Baseado nessas consideraçôes, a Fisioterapia em Oncologia visa a prevenir e/ou controlar possíveis manifestaçôes pós-operatórias e/ou radioterapêuticas, sendo fundamental atuar de forma precoce em equipes multidisciplinares. A reeducação do membro superior é uma necessidade básica dessa paciente, independente da técnica cirúrgica. Nesse sentido, o tratamento fisioterapêutico tem o intuito de restabelecer os movimentos, despertar o sentimento de independência e estimular a sua percepção da importância da qualidade de vida ${ }^{1}$.

Dessa forma, o presente estudo objetiva verificar o impacto da aplicação de um protocolo fisioterapêutico sobre a funcionalidade e a qualidade de vida de mulheres com câncer de mama alocadas em uma casa de apoio a pacientes oncológicos em Uruguaiana (RS).

\section{Método}

Trata-se de um estudo experimental, autocontrolado, de forma descritiva e analítica em uma abordagem antes-depois ${ }^{7}$, com o principal objetivo de verificar os efeitos de protocolo fisioterapêutico sobre a funcionalidade do membro superior e a qualidade de vida, realizado em uma casa de apoio a pacientes oncológicos de Uruguaiana (RS) mediante autorização da direção da instituição.

O universo populacional foi constituído por mulheres com diagnóstico de câncer de mama que eram participantes da instituição, sendo que, para a confecção da amostra, usamos os critérios de inclusão e exclusão dispostos na Figura 1 (organograma), além do aceite do Termo de Consentimento Livre e Esclarecido (TCLE).

Figura 1: Organograma dos critérios de inclusão do estudo.

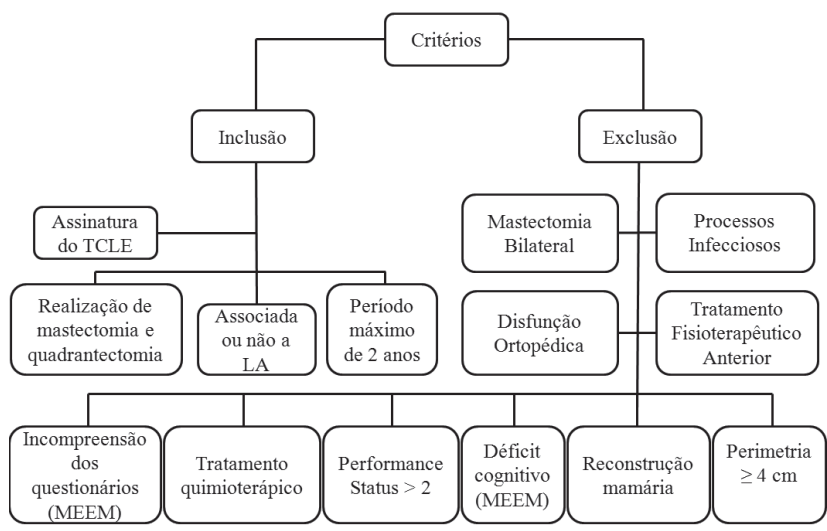

Legenda: LA = linfadenectomia axilar, TCLE $=$ termo de consentimento livre e esclarecido, $M E E M=$ miniexame do estado mental.

O presente projeto foi aprovado pelo Comitê de Ética e Pesquisa (CEP) da instituição sob o no 10.027.14.

Utilizamos o Programa Epi Info Versão 7.0 para calcular o tamanho da amostra considerando a qualidade de vida como desfecho, com base em outra pesquisa ${ }^{8}$ com poder de $80 \%$ e nível de significância de $5 \%$, resultando em 43 indivíduos.

Quanto aos procedimentos, as voluntárias foram submetidas às avaliações:

1. Miniexame do estado mental (MEEM) para avaliação do status cognitivo, utilizando como pontos de corte para déficit analfabetos $\leq 15$ pontos, 1 a 11 anos de escolaridade $\leq 22$ e com escolaridade superior a 11 anos $\leq 27^{9}$.

2. Escala de capacidade funcional por meio do Eastern Cooperative Oncology Group (ECOG) Performance Status: investiga o estado funcional do paciente oncológico, considerando a qualidade de vida e as atividades de vida diária cujos escores são entre 0 e 4 , em que 0 representa paciente com atividade normal; destaca-se que ambas as avaliações, MEEM e ECOG, tiveram como objetivo a seleção de voluntárias.

3. Avaliaçáo geral: com dados pessoais como nome, idade, etnia e ocupação; história clínica 
e pregressa como tipo de intervenção cirúrgica, tratamentos conservadores, ressecção linfonodal, complicaçôes físico-funcionais e doenças ortopédicas-reumatológicas; dados uroginecológicos como menarca, gestação, amamentação e menopausa; hábitos e estilo de vida como atividade física e uso de anticoncepcionais.

4. Perimetria para avaliar a presença de linfedema, que foi medida com o auxílio de uma fita métrica (fiberglass) padronizada em centímetros $(\mathrm{cm})$, sendo a voluntária posicionada em sedestação com o membro superior ao longo do corpo. Utilizamos como pontos de marcação a linha articular do cotovelo e o olécrano (ponto zero), mensurando 5, 10, 15 e $20 \mathrm{~cm}$ supra e infraolécrano, demarcados com lápis dermatográfico, tendo como referência o membro superior contralateral ${ }^{10}$.

5. A goniometria, para fins de avaliação da amplitude de movimento, foi realizada conforme os parâmetros já descritos ${ }^{11}$, utilizando o goniômetro (ISP), em que se compara o membro superior (MMSS) homolateral ao contralateral com a voluntária em sedestação e bipedestação nos movimentos de flexão, extensão, abdução, adução, rotação interna e rotação externa de ombro.

6. Questionário de qualidade de vida SF-36: composto por 36 itens, que englobam capacidade funcional, aspectos físicos, dor, estado geral da saúde, vitalidade, aspectos sociais, aspectos emocionais e saúde mental. Os escores variam de 0 a 100 , no qual zero corresponde ao pior estado geral de saúde, e 100, ao melhor $^{12}$.

Após as avaliaçóes, as participantes, de forma coletiva, foram submetidas a um protocolo de intervenção fisioterapêutico ${ }^{13}$, com periodicidade de 10 sessōes, duraçáo de 1 hora, duas vezes por semana, constituído de: mobilização da cicatriz mamária; alongamento dos grupos musculares - coluna cervical e região escapular; flexores e extensores de punho; flexores e extensores de ombro, peitorais e rotadores externos de ombro com uso de um bastão; exercícios ativo-livres em todos os planos de movimento dos membros superiores (flexáo, extensão, abdução, adução, rotaçôes interna e externa) utilizando bastão, faixa elástica e bola, com séries de 15 repetiçôes para cada exercício (Figura 2) e orientaçóes domiciliares quanto ao cuidado com a pele e com a higiene do membro superior, realizaçáo de movimentos do membro superior homolateral em casa, nas atividades de trabalho e de lazer, por meio de uma cartilha elaborada pelas pesquisadoras.
Para a análise dos dados, foram avaliadas as frequências absoluta (n) e relativa (\%) de cada variável e/ou a média \pm desvio padrão.

Figura 2: Protocolo fisioterapêutico.

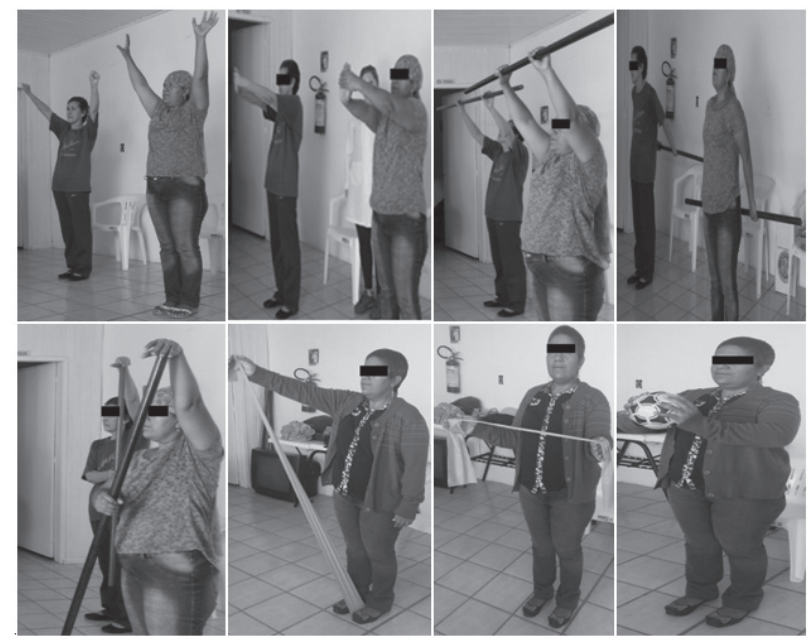

Legenda: $\boldsymbol{a}$ - exercício livre de abdução de ombro favorecendo bombeamento venoso; $\boldsymbol{b}$ - alongamento ativo de flexores de punho; $\boldsymbol{c}$ - exercício ativo de flexão de ombro utilizando bastão; $\boldsymbol{d}$ - exercício ativo de extensão de ombro; $\boldsymbol{e}$ - exercício ativo de abdução de ombro utilizando bastáo; $f$ - exercício resistido de flexores e abdutores de ombro com faixa elástica; $g$ - exercício resistido de rotadores externos de ombro utilizando faixa elástica; $\boldsymbol{b}$ - exercício resistido de adutores de ombro utilizando bola.

\section{Resultados}

Inicialmente, foram incluídas seis participantes. Contudo, houve perda amostral de duas pacientes por problemas pessoais. Como resultado, quatro voluntárias na faixa etária de 44 a 64 anos concluíram o protocolo fisioterapêutico, três $(75 \%)$ foram submetidas à mastectomia associada à radioterapia (Tabela 1).

Os resultados referentes à amplitude de movimento antes e após o protocolo estão expostos no Quadro 1, sendo possível observar que todas as voluntárias, inclusive as na faixa etária dos 60 anos, obtiveram melhora no grau de amplitude de movimento quando comparado com os resultados anteriores ao protocolo de exercícios, especialmente nos movimentos de flexão e abdução de ombro, que são os mais restritos no pós-operatório de mama, com exceção de uma paciente, que manteve o mesmo grau de rotação interna de ombro após as 10 sessôes de fisioterapia. Associadamente, nenhuma voluntária apresentou linfedema, mensurado pela perimetria realizada na avaliação e reavaliação, nem previamente à intervenção fisioterapêutica nem após o protocolo de 10 sessóes. 
Tabela 1: Características clínicas das participantes.

\begin{tabular}{|c|c|}
\hline $\begin{array}{l}\text { Idade das participantes } \\
\text { Perfil clínico das participantes }\end{array}$ & $54 \pm 11,5$ anos \\
\hline \multicolumn{2}{|l|}{ Etnia } \\
\hline Branca & $3(75 \%)$ \\
\hline Parda & $1(25 \%)$ \\
\hline \multicolumn{2}{|l|}{ Ocupação } \\
\hline Doméstica & $2(50 \%)$ \\
\hline Funcionária pública & $1(25 \%)$ \\
\hline Auxiliar de enfermagem & $1(25 \%)$ \\
\hline \multicolumn{2}{|l|}{ Dados uroginecológicos } \\
\hline \multicolumn{2}{|l|}{ Status hormonal } \\
\hline Menopausa fisiológica & $3(75 \%)$ \\
\hline Ciclo menstrual normal & $1(25 \%)$ \\
\hline \multicolumn{2}{|l|}{ Reposiçáo hormonal } \\
\hline Não & $4(100 \%)$ \\
\hline Sim & - \\
\hline \multicolumn{2}{|l|}{ Tipos de cirurgia } \\
\hline Mastectomia total & $3(75 \%)$ \\
\hline Quadrantectomia + linfadenectomia axilar & $1(25 \%)$ \\
\hline \multicolumn{2}{|l|}{ Tratamento associado } \\
\hline Radioterapia & $3(75 \%)$ \\
\hline Quimioterapia & $1(25 \%)$ \\
\hline \multicolumn{2}{|l|}{ Tempo entre cirurgia e início da fisioterapia } \\
\hline$<1$ ano & $1(25 \%)$ \\
\hline$<2$ anos & $3(75 \%)$ \\
\hline \multicolumn{2}{|l|}{ Comorbidades associadas } \\
\hline Hipertensão arterial & $3(75 \%)$ \\
\hline Osteoartrite de joelhos & $1(25 \%)$ \\
\hline \multicolumn{2}{|l|}{ Status cognitivo (MEEM) } \\
\hline Pontuação: 30 & $2(50 \%)$ \\
\hline Pontuação: 28 & $1(25 \%)$ \\
\hline Pontuação: 26 & $1(25 \%)$ \\
\hline \multicolumn{2}{|l|}{ Status funcional (performance status) } \\
\hline Pontuação: 1 & $4(100 \%)$ \\
\hline
\end{tabular}

Quadro 1: Amplitudes de movimento pré e pós-protocolo fisioterapêutico.

\begin{tabular}{|c|c|c|c|c|c|c|c|c|c|c|c|c|c|}
\hline \multirow[t]{2}{*}{ Voluntárias } & \multirow[t]{2}{*}{$\begin{array}{c}\text { Membro } \\
\text { acometido }\end{array}$} & \multicolumn{2}{|c|}{ Flexáo } & \multicolumn{2}{|c|}{ Extensão } & \multicolumn{2}{|c|}{ Abdução } & \multicolumn{2}{|c|}{ Adução } & \multicolumn{2}{|c|}{$\begin{array}{c}\text { Rotaçáo } \\
\text { Interna }\end{array}$} & \multicolumn{2}{|c|}{$\begin{array}{c}\text { Rotaçáo } \\
\text { externa }\end{array}$} \\
\hline & & Pré & Pós & Pré & Pós & Pré & Pós & Pré & Pós & Pré & Pós & Pré & Pós \\
\hline A & Direito & $140^{\circ}$ & $152^{\circ}$ & $40^{\circ}$ & $45^{\circ}$ & $150^{\circ}$ & $160^{\circ}$ & $40^{\circ}$ & $40^{\circ}$ & $84^{\circ}$ & $90^{\circ}$ & $85^{\circ}$ & $88^{\circ}$ \\
\hline B & Esquerdo & $130^{\circ}$ & $140^{\circ}$ & $54^{\circ}$ & $70^{\circ}$ & $144^{\circ}$ & $146^{\circ}$ & $30^{\circ}$ & $30^{\circ}$ & $80^{\circ}$ & $90^{\circ}$ & $84^{\circ}$ & $90^{\circ}$ \\
\hline $\mathrm{C}$ & Direito & $120^{\circ}$ & $150^{\circ}$ & $30^{\circ}$ & $40^{\circ}$ & $110^{\circ}$ & $130^{\circ}$ & $20^{\circ}$ & $40^{\circ}$ & $70^{\circ}$ & $85^{\circ}$ & $0^{\circ}$ & $60^{\circ}$ \\
\hline $\mathrm{D}$ & Esquerdo & $124^{\circ}$ & $150^{\circ}$ & $42^{\circ}$ & $66^{\circ}$ & $140^{\circ}$ & $156^{\circ}$ & $30^{\circ}$ & $46^{\circ}$ & $90^{\circ}$ & $90^{\circ}$ & $48^{\circ}$ & $52^{\circ}$ \\
\hline
\end{tabular}

No Quadro 2, são demonstrados os resultados referentes à qualidade de vida das participantes por meio do SF-36. As voluntárias apresentaram melhora, principalmente na capacidade funcional, estado geral em saúde, vitalidade, aspectos sociais, aspectos emocionais e saúde mental. Entretanto, duas (50\%) alegaram piora no domínio da dor, tendo-se como possíveis justificativas a osteoartrite de joelhos e pelos efeitos do tratamento farmacológico para alergia, como indisposição e fraqueza. Uma participante $(25 \%)$ demonstrou piora no aspecto físico, o que pode ser explicado pelo fato de estar realizando radioterapia concomitantemente ao protocolo fisioterapêutico.
Quadro 2: Qualidade de vida prée pós-protocolo fisioterapêutico.

\begin{tabular}{|c|c|c|c|c|c|c|c|c|}
\hline Voluntárias & \multicolumn{2}{|c|}{$\mathbf{A}$} & \multicolumn{2}{|c|}{ B } & \multicolumn{2}{|c|}{$\mathrm{C}$} & \multicolumn{2}{|c|}{ D } \\
\hline Domínios & Pré & Pós & Pré & Pós & Pré & Pós & Pré & Pós \\
\hline $\begin{array}{l}\text { Capacidade } \\
\text { funcional }\end{array}$ & 60 & 70 & 35 & 90 & 55 & 90 & 50 & 85 \\
\hline Aspectos físicos & 0 & 100 & 25 & 100 & 75 & 100 & 75 & 25 \\
\hline Dor & 72 & 100 & 100 & 84 & 72 & 84 & 72 & 52 \\
\hline $\begin{array}{l}\text { Estado geral de } \\
\text { saúde }\end{array}$ & 37 & 62 & 57 & 80 & 72 & 87 & 62 & 62 \\
\hline Vitalidade & 90 & 90 & 45 & 70 & 75 & 90 & 90 & 90 \\
\hline Aspectos sociais & 97,5 & 100 & 0 & 100 & 100 & 100 & 100 & 100 \\
\hline $\begin{array}{l}\text { Aspectos } \\
\text { emocionais }\end{array}$ & 0 & 100 & 0 & 100 & 66,6 & 100 & 100 & 100 \\
\hline Saúde mental & 84 & 92 & 80 & 96 & 84 & 96 & 84 & 100 \\
\hline
\end{tabular}




\section{Discussão}

A fisioterapia realizada após a intervenção cirúrgica de câncer de mama objetiva o retorno da paciente às suas funçóes (atividades), com um menor grau de limitação residual, sendo um dos recursos fisioterapêuticos mais utilizados na cinesioterapia, em acordo com a proposta de duas pesquisas: a primeira, de 2008, considera tal recurso fundamental para a reabilitação física, melhorando a recuperação funcional do membro e prevenindo complicações, como linfedema, alteraçôes posturais, encurtamentos musculares, retração e aderência cicatricial ${ }^{14}$; a segunda, de 2009 , destaca que a limitação articular homolateral à cirurgia deve ser prevenida e/ou tratada o mais precocemente possível ${ }^{15}$.

A cinesioterapia como recurso fisioterapêutico apresenta possibilidades de atuação conforme as principais necessidades elencadas pelas voluntárias. Dessa forma, geralmente, as mulheres submetidas a ressecções cirúrgicas para o câncer de mama apresentam limitaçóes articulares na flexão, abdução e rotação externa ${ }^{16}$. Nesse sentido, o mesmo autor e colaboradores elaboraram um protocolo com alongamentos na região cervical, escápula, membros superiores e exercícios ativos resistidos durante 8 semanas para 10 mulheres na faixa etária de 47 a 49 anos, obtendo resultados significativos quanto à amplitude de movimento de flexão, extensão, abdução e rotação interna do ombro homolateral. Os movimentos de flexão e abdução são essenciais para a funcionalidade do membro superior, fato que retrata a importância do desse estudo.

É importante considerar ainda que distintos fatores podem influenciar nos arcos de movimento, como a dimensão e o aspecto da cicatriz cirúrgica, a presença de dor e o receio de movimentar o membro superior ipsilatera ${ }^{13}$. Essas características foram avaliadas em um protocolo cinesioterapêutico envolvendo a articulação do ombro associado à mobilização cicatricial, por 10 sessóes, em 10 mulheres com faixa etária de 41 a 59 anos, que melhoraram a amplitude de movimentos das participantes e, consequentemente, sua qualidade de vida $^{13}$, em consonância com o presente estudo. Além do nosso, outro estudo também mostrou que a mobilização cicatricial estimula a propriocepção, favorecendo, assim, a amplitude de movimento ${ }^{17}$.

Outro protocolo cinesioterapêutico englobou 20 sessóes contemplando alongamentos da região cervical e membros superiores, exercícios ativos livres envolvendo todos os movimentos de ombro, aplicados de maneira progressiva e conduzidos por duas pesquisadoras como no presente estudo, em que a partir da $6^{a}$ sessão fisioterapêutica, conforme a evolução das participantes, os exercícios eram complementados com faixas elásticas e bolas ${ }^{18}$. No presente estudo, similarmente à pesquisa supracitada, houve um aumento na amplitude de movimento do ombro, tendo como distinção o tempo do protocolo; todavia, há evidências de que um mínimo de 10 sessóes do protocolo fisioterapêutico já reduz o quadro álgico e aumenta os arcos, refletindo na qualidade do movimento e na realização das tarefas diárias ${ }^{18}$.

Associadamente à cinesioterapia, a adesão à realização dos protocolos é essencial para que haja impacto positivo sobre a funcionalidade, considerando que existem fatores interferentes, como a presença de comorbidades, as crenças pessoais e o acesso ao tratamento. Nesse sentido, no presente estudo a maioria das participantes aderiu à proposta, em concordância com um estudo semelhante ${ }^{19}$, que sinaliza que experiências em grupo apresentam efeito positivo tanto no âmbito físico quanto emocional, colaborando, assim, para a adesão.

Do ponto de vista da satisfação com sua saúde, ilustrado pela evolução da capacidade funcional, as participantes do presente estudo relataram maior facilidade nas atividades de vida diária, similarmente à outra pesquisa, a qual contou com uma amostra de 11 mulheres, sendo 7 mastectomizadas e 4 que realizaram quadrantectomia, com idade média de 56,6 anos, em pós-operatório tardio e voluntárias submetidas a exercícios ativos resistidos, alongamentos, autodrenagem do membro superior homolateral e a cirurgia. Esse tratamento foi feito uma vez na semana, por um período de 12 semanas, relatando ao final do estudo melhora na disposição, no ânimo, na energia e no bem-estar, com a prática de exercícios físicos ${ }^{20}$.

Mulheres mastectomizadas e/ou em tratamento quimioterápico e/ou radioterápico são submetidas a altos níveis de ansiedade e a quadros depressivos por enfrentarem sua(s) doença(s), piorando sua qualidade de vida. Dessa forma, são propostas terapias em grupo, proporcionando trocas de experiências, aumento do ânimo, energia e bem-estar, além dos ganhos físico-funcionais, em acordo com os achados do presente estudo ${ }^{21}$.

Faz-se importante reforçar que distintos aspectos comprometem a qualidade de vida de mulheres mastectomizadas, incluindo limitação da mobilidade do membro superior, tratamentos adjuvantes e sintomas como dor e linfedema, bem como distorção da autoimagem, quadros de depressão e ansiedade, que de forma associada ou náo, tendem a repercutir sobre o processamento saúde-adoecimento $^{22}$. Com base nessas consideraçóes e nos achados do presente estudo, após a aplicação do protocolo fisioterapêutico, as participantes obtiveram melhora na maioria dos domínios referentes à qualidade de vida, sinalizando um impacto geral sobre a saúde de cada voluntária.

\section{Conclusão}

Verificamos que a aplicaçáo do protocolo fisioterapêutico de curta duração ampliou os movimentos do 
ombro homolateral, melhorando a qualidade de vida das participantes. Entretanto, os resultados não podem ser extrapolados para a populaçáo em geral, pois foi um estudo realizado em uma casa de apoio a pacientes oncológicos, além do número limitado de voluntárias participantes. Contudo, esperamos que esses resultados possam contribuir para aprofundar as terapias do câncer de mama, especialmente em caráter longitudinal.

\section{Referências}

1. Jammal MP, Machado ARM, Rodrigues LR. Fisioterapia na reabilitação de mulheres operadas por câncer de mama. O Mundo da Saúde 2008;4(32):506-10.

2. Guirro E, Guirro R. Fisioterapia dermato-funcional: fundamentos, recursos, patologias. $3^{\mathrm{a}} \mathrm{ed}$. Barueri (SP): Manole; 2004.

3. Velloso FSB, Barra AA, Dias RC. Morbidade de membros superiores e qualidade de vida após a biópsia de linfonodo sentinela para o tratamento de câncer de mama. Rev Bras Cancerol 2009;55(1):75-85.

4. Quinto SMG, Mejia DPM. Benefícios da fisioterapia no tratamento de linfedema pós-mastectomia radical: uma revisão literária. [tese de doutorado]. Goiânia: Faculdade Ávila; 2012 [acesso em 2015 abr 11]. Disponível em: http:// scholar.googleusercontent.com/scholar?q=cache:JeGsIiHtM7gJ:scholar.google.com/\&hl=en\&as_sdt=0,5\&as_vis=1

5. Gusmão C. Drenagem linfática manual: método Dr. Vodder. $1^{\mathrm{a}}$ ed. Sáo Paulo: Atheneu; 2010.

6. Rezende LF, Beletti PO, Franco RL, Moraes SS, Gurgel MSC. Exercícios livres versus direcionados nas complicaçôes pós-operatórias de câncer de mama. Rev Assoc Med Bras 2006;1(52):37-42.

7. Marconi MA, Lakatos EM. Fundamentos de metodologia científica. 5a ed. São Paulo: Atlas; 2003.

8. Silva MD, Rett MT, Mendonça ACR, Junior WMS, Prado VM, Santana JM. Qualidade de vida e movimento do ombro no pós-operatório de câncer de mama: um enfoque da fisioterapia. Rev Bras Cancerol 2013;59(3):419-26.

9. O’keeffe ST, Mulkerrin EC, Naveem K, Varughese M, Pillay I. Use of serial mini-mental state examinations to diagnose and monitor delirium in elderly hospital patients. J Amer Geriatr Soc 2005;53(5):867-70.

10. Levone BR, Schossler MS, Pedrini A, Silva IS. Fisioterapia no pré e pós-operatório imediato de cirurgia de quadrantectomia mamária: um estudo de caso. EFDeportes 2011;15(152):1.

11. Marques AP. Manual de goniometria. $3^{\mathrm{a}}$ ed. Barueri: Manole; 2003.

12. Ciconelli RM, Ferraz MB, Santos W, Meinão I, Quaresma MR. Tradução para a língua portuguesa e validação do questionário genérico de avaliação de qualidade de vida SF-36 (Brasil SF-36). Rev Bras Reumatol 1999;39(3):143-50.

13. Rett MT, Santos AKG, Mendonça ACR, Oliveira IA, Santana JM. Efeito da fisioterapia no desempenho funcional do membro superior no pós-operatório de câncer de mama. Rev Ciênc Saúde 2013;6(1):18-24.

14. Almeida SP. A cinesioterapia em paciente pós-mastectomizada. [Monografia]. Rio de Janeiro: Universidade Veiga de Almeida; 2008 [acesso em 2015 jun 15]. Disponível em: https:/www.uva.br/sites/all/themes/uva/files/pdf/a_cinesiologia_em_paciente_pos_mastectomizada.pdf

15. Silva SH, Godoy JMP. Avaliação da amplitude de movimento de ombro. Acta Med Port 2009;5(22):567-70.

16. Leites GT, Knorst MR, Lima CHL, Zerwes FP, Friso VB. Fisioterapia em oncologia mamária: qualidade de vida e evolução clínico funcional. Rev Ciênc Saúde 2010;3(1):14-21.

17. Nascimento SL, Oliveira RR, Oliveira MMF, Amaral MTP. Complicaçôes e condutas fisioterapêuticas após cirurgia por câncer de mama: estudo retrospectivo. Rev Fisioter Pesqui 2012;19(3):248-55.

18. Rett MT, Mesquita PJ, Mendonça ARC, Moura DP, Santana JM, A cinesioterapia reduz a dor no membro superior de mulheres submetidas à mastectomia ou quadrantectomia. Rev Dor 2012;13(3):201-7.

19. Gutiérrez MGR, Bravo MM, Chanes DC, Vivo MCR, Souza GO. Adesão de mulheres mastectomizadas ao início precoce de um programa de reabilitaçáo. Acta Paul Enferm 2007;20(3):249-54.

20. Pischel GCF, Graziani SR. Programa de fortalecimento muscular direcionado a pacientes portadores de carcinoma de mama no pós-operatório tardio. Rev Pibic 2006;3(2):79-86.

21. Tacani PM, Baptista PAN, Campos CM, Kasawara KT, Gimenes RO. Fisioterapia em grupo na reabilitação funcional dos membros superiores de mulheres pós-mastectomia. Ter Man 2013;51(11):1-6.

22. Lahoz MA, Nyssen SM, Correia GN, Garcia APU, Driusso P. Capacidade funcional e qualidade de vida em mulheres pós-mastectomia. Rev Bras Cancerol 2010;56(4):423-30.

\section{Como citar este artigo:}

Nava LP, Martins CF, Lara S, Ferreira FV. Fisioterapia em oncologia mamária. Rev. Aten. Saúde. 2016;14(48):21-26. 